\title{
Lower blepharoplasty: how we do it
}

\author{
Chuan-Hsiang Kao, MD ${ }^{1,2}$ (iD, Sarina Rajbhandari, $\mathrm{MD}^{3}$ (iD \\ 'Department of Otolaryngology-Head and Neck Surgery, Tri-Service General Hospital, National Defense Medical Center, Taipei, Taiwan, Rep. of \\ China \\ ${ }^{2}$ Hope Aesthetic Clinic, Taipei, Taiwan, Rep. of China \\ ${ }^{3}$ Shankarapur Academy and Hospital, Kathmandu, Nepal
}

The natural process of ageing causes multiple age-related changes, which are observed in the anatomy of the eyelids and surrounding structures, including the malar region. Blepharoplasty is a procedure performed widely for tired looking eyes and facial rejuvenation. Lower blepharoplasty (LBP) is usually done with fat transposition rather than fat resection to avoid hollow appearance of malar area. Patients with excess fat and no tear trough deformity can be treated with fat resection alone, where as those with a prominent tear trough deformity requires fat transposition. Transposing the medial and central fat pads instead of excising them can help to fill out the hollowness of the under eye area whereas the lateral fat pads are removed as much as needed through direct excision. In our practice, we generally prefer a transconjunctival approach to lower lid blepharoplasty with fat repositioning.

Keywords: aging; lower blepharoplasty; transconjunctival

\section{Introduction}

Aging causes multiple age-related changes in the eyelids and surrounding structures, including the malar region. The concomitant loss of elasticity of the musculature, and overlying skin, resulting in a saggy appearance, of the inferior eyelids contribute significantly to the elongation of the vertical aperture, increasing the visibility of the sclera [1]. A bulge develops under the eyes due to excess skin, muscle, and fat, which causes an unattractive and aged appearance, as opposed to a youthful face, which has a natural rounding and projection of the cheek, an elevated malar fat pad position, inconspicuous tear trough, and less pronounced nasolabial folds.

The term "blepharoplasty" was coined by Von Graefe in 1818 [2]. The first transcutaneous approach to retroseptal fat was performed by Sir Archibald McIndoe using skin-muscle flap. Fat preservation and translocation for smooth transition between the lower eyelid and cheek were introduced by Loeb [3].

Blepharoplasty is performed widely for tired-looking and ageing eyes. It plays a key role in facial rejuvenation. The two main concerns in blepharoplasty are the excess fat or malposition of fat and excess eyelid skin [4]. For lower eyelid rejuvenation, the main goal is to create a smooth contour from the lower eyelid skin to the cheek by

- Removing the redundant skin

- Smoothening out and tightening the underlying tissues and muscle

- Repositioning or removing the excess fat

In our practice, we prefer a transconjunctival approach to lower eyelid blepharoplasty with fat repositioning. Good candidates for this approach include young or middle-aged people with mild to moderate drooping of the periorbital tissue and

Received December 9, 2019; Revised December 28, 2019; Accepted December 30, 2019

Corresponding author: Chuan-Hsiang Kao

E-mail: chuanhsiang@yahoo.com.tw

This is an Open Access article distributed under the terms of the Creative Commons Attribution Non-Commercial License (http://creativecommons.org/licenses/by-nc/4.0), which permits unrestricted non-commercial use, distribution, and reproduction in any medium, provided the original work is properly cited.

Copyright ( 2019 Korean Society of Korean Cosmetic Surgery and Medicine (KSKCS \& KCCS). 
indistinct redundancy of the lower eyelid skin.

\section{Transconjunctival approach}

The transconjunctival approach to lower eyelid blepharoplasty started as early as in the late 1920s. The landmark contribution that made this procedure more widely acceptable was that by Zarem and Resnick [5] in 1993.

The classic concept of Castanares [6] involved orbital fat excision as the primary approach to lower eyelid bulging. Overresection of fat left a hollowed-out appearance, creating a more aged appearance.

Lower eyelid blepharoplasty is usually performed with fat transposition rather than fat resection to avoid a hollow appearance of the malar area. Transposing native eyelid fat to the nasojugal groove (tear trough) has become a standard adjunct to lower eyelid blepharoplasty in the appropriate patient [7]

\section{Preoperative evaluation}

General history taking and examination are performed before surgery. Medical conditions such as hypertension, coagulation disorders, and previous eye surgeries are considered before performing lower eyelid blepharoplasty. Clinical examination involves determining the degree of lower eyelid laxity, orbicularis oculi muscle tone, presence or absence of the nasojugal groove, excess skin or fat, contour of the lower eyelid-cheek interface, and asymmetries of the eyelid or orbit [4]. Lower eyelid laxity is assessed using snap and distraction tests.

Surgical procedures are designed to preserve eyelid function and prevent malposition. Patients with excess fat and no tear trough deformity can be treated with fat resection alone. Those with a prominent tear trough deformity with depression require fat transposition.

The surgeon should also explain to the patient that lower eyelid blepharoplasty is not meant to address fine skin wrinkling.

\section{Surgical procedure}

Transconjunctival blepharoplasty is routinely performed with local or preferably intravenous anesthesia (IVA) and can be performed in an office operating room setting. The conjunctiva and cornea are anesthetized with 2 drops of $0.5 \%$ tetracaine hydrochloride ophthalmic solution instilled into the lower fornix of each eye. We then inject a local anesthetic solution (1\%-2\% lidocaine with 1:100,000 epinephrine) in the lower fornix using a 30-gauge needle as the transconjunctival injection. We use corneal protectors during the surgery to protect the eyeball and prevent drying of the cornea. We also place stay sutures through the medial and lateral conjunctival surfaces of the inferior fornix to retract the conjunctiva superiorly, which also protects the cornea. The lower eyelid margin is retracted with a small double hook to expose the inferior fornix for a safe dissection (Fig. 1).

\section{Incision}

An incision is made with a Colorado tip monopolar cautery in the conjunctiva, approximately $4 \mathrm{~mm}$ below the tarsus, close to the fornix, extending from the lacrimal caruncle to near the lateral canthus. We use a Desmarres retractor for caudal retraction to create a better visual field. We then identify the orbital septum with the fat compartments.

\section{Dissection}

Dissection is continued while obtaining hemostasis. We also use Q-tips with gentle sweeping motions to proceed with our dissection. The Desmarres retractor is then replaced with the
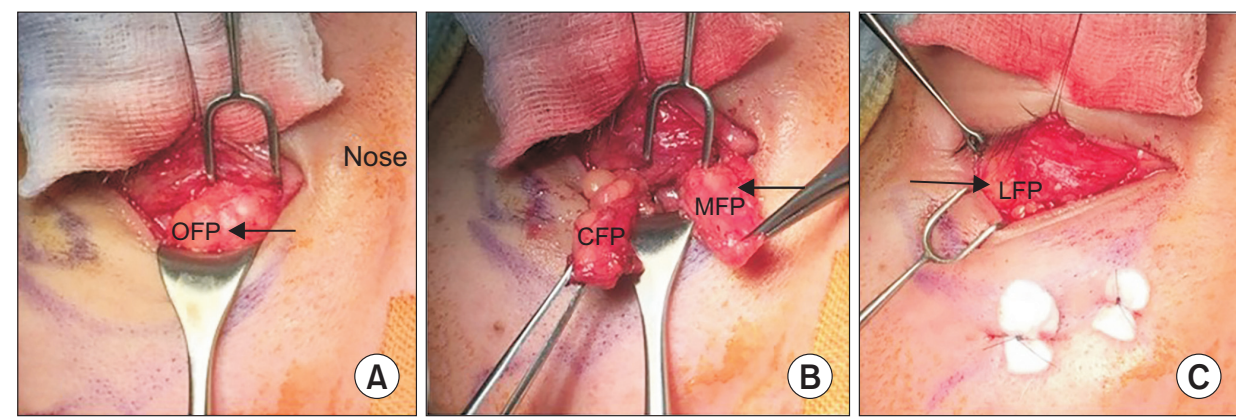

Fig. 1. Intraoperative view of the right eye. (A) Trans-conjunctival pre-septal dissection was performed to expose the orbital fat (arrow). (B) The medial (arrow) and central fat pads (pull dowanwards) were well dissected and released. (C) Transcutaneous stay sutures on downwards repositioned medial and central fat pads with 4-O nylon. The lateral fat pad (arrow) can be partially excised to prevent postoperative bulging. OFP, orbital fat pad; MFP, medial fat pad; CFP, central fat pad; LFP, lateral fat pad. 
blunt end of the cat's paw retractor to look for the arcus marginalis, a whitish line slightly cranial to the origin of the palpebral part of the orbicularis oculi. The tear trough ligament, palpebral and orbital parts of the orbicularis oculi, and medial part of the orbicularis-retaining ligament are released, keeping the dissection close to the anterior maxilla. Initially, we dissect at the subperiosteum level and then perform a supraperiosteal dissection if necessary.

\section{Transseptal approach to orbital fat}

We apply the direct technique by incising through the septum, exposing the fat compartments for transposition. Gentle continuous pressure is applied over the globe to allow the fat to herniate through the incised septum. The medial fat pad, which is paler in appearance, is assessed first. We then inject local anesthetic mixture to the fat and its pedicle. The fat capsule is incised so that the underlying fat spills out. The fat is dissected up to its pedicle or as far as we can go. The central fat pad is more yellow, and a similar technique is applied to it.

In supraperiosteal redraping, the suborbicularis oculi fat (SOOF) is identified and blunt dissection is performed on the lateral to infraorbital neurovascular bundle to create a pocket for redraping. The medial and central fat pads are held with fine forceps, moving them around the inferior oblique muscle in a reverse shoe-shining action. A 4-O nylon suture is brought in through the skin, through the medial fat pad, descending downward, inferior to the tear trough. The needle is brought out transcutaneously through the skin and sutured, placing a small bolster of cotton on the skin to avoid irritation. Similarly, the central fat pad is also sutured inferolaterally or to the hollows of the lower eyelids, which are marked before surgery. The lateral fat pad is allowed to bulge by applying slight pressure on the globe and then excised. To avoid hematoma, the base of the fat pads should be cauterized before they retract back quickly when released.

The conjunctival incision is then closed with three to four interrupted 7-0 Vicryl fast resorbable sutures. Postoperatively, the patient is given antibiotic ophthalmic solutions and cold compress over the lower eyelids. We remove the cutaneous sutures after 3 to 5 days.

\section{Discussion}

The lower eyelid blepharoplasty technique described in this report is aimed at achieving a better aesthetic outcome in patients with hollow eyelids or hypoplastic malar eminences.
Postoperative ectropion and reduced scarring is often seen in transconjunctival approach. The malar complex must be considered part of the lower eyelid during lower eyelid blepharoplasty to achieve satisfactory, youthful results. A smooth transition in the lower eyelid-cheek junction must be obtained as much as possible. In revision cases or cases with a severe hollow midface, autogenous facial fat transplantation may be considered to achieve more favorable results. Transposing the medial and central fat pads instead of excising them can help fill up the hollowness of the area under the eyes, whereas the lateral fat pads are removed as much as needed.

In a retrospective study that involved 40 patients with a mean follow up of 10 months, Yoo et al. [8] found that in transconjunctival lower eyelid blepharoplasty, the ultimate aesthetic results of fat transposition to either the subperiosteal or supraperiosteal plane were comparable, but supraperiosteal transposition caused greater trauma, including more bruising and swelling.

The common complications include swelling, chemosis, ecchymosis, conjunctiva adhesion, transient diplopia, entropion, and lagophthalmos. Uncontrolled bleeding within the orbital fat compartment may lead to retrobulbar hemorrhage. Thus, while excising fat, hemostasis is obtained before releasing the base/pedicle of the fat pad as it retracts back quickly. Any severe eye pain or decrease in vision is considered an emergency.

When patients exhibit excess low eyelid skin, a skin flap can be elevated through a subciliary incision and skin can be excised. By using this method, the underlying orbicularis muscle is not violated. The extended transconjunctival technique by dissecting through the facial soft tissue spaces of the mid cheek with precise release of the retaining ligaments that separate them is effective in rejuvenating the upper mid cheek, with correction of tear trough deformity and eye bags while minimizing bruising, swelling, and downtime [9].

\section{Conclusion}

Transconjunctival approach to lower eyelid blepharoplasty with fat repositioning is a safe approach with a pleasant cosmetic outcome for patients without severe orbital skin laxity.

\section{Conflicts of interest}

The authors have nothing to disclose. 


\section{References}

1. Chen JL, Liu CH, Yi CG. Indications of transconjunctival orbital septum fat release and preservation for orbitopalpebral sulcus in lower eyelid blepharoplasty. Aesthetic Plast Surg 2017;41:753.

2. Dupuis C, Rees TD. Historical notes on blepharoplasty. Plast Reconstr Surg 1971;47:246-51.

3. Loeb R. Fat pad sliding and fat grafting for leveling lid depressions. Clin Plast Surg 1981;8:757-76.

4. Davison SP, Iorio ML, Oh C. Transconjunctival lower lid blepharoplasty with and without fat repositioning. Clin Plast Surg 2015;42:51-6.

5. Zarem HA, Resnick JI. Minimizing deformity in lower blepharoplasty. The transconjunctival approach. Clin Plast Surg 1993;20:317-21.
6. Castanares S. Blepharoplasty for herniated intraorbital fat; anatomical basis for a new approach. Plast Reconstr Surg (1946) 1951;8:46-58.

7. Massry GG, Nassif PS. Transconjunctival lower blepharoplasty: fat excision or repositioning. In: Massry GG, Murphy MR, Azizzadeh B, editors. Master techniques in blepharoplasty and periorbital rejuvenation. New York (NY): Springer; 2011. p. 173-84.

8. Yoo DB, Peng GL, Massry GG. Transconjunctival lower blepharoplasty with fat repositioning: a retrospective comparison of transposing fat to the subperiosteal vs supraperiosteal planes. JAMA Facial Plast Surg 2013;15:176-81.

9. Wong $\mathrm{CH}$, Mendelson B. Extended transconjunctival lower eyelid blepharoplasty with release of the tear trough ligament and fat redistribution. Plast Reconstr Surg 2017;140:273-82. 